\title{
BMJ Open Effectiveness of prehabilitation for patients undergoing orthopaedic surgery: protocol for a systematic review and meta-analysis
}

\author{
Anuj Punnoose (D) , ${ }^{1,2}$ Ori Weiss, ${ }^{3}$ Vikas Khanduja, ${ }^{3}$ Alison B Rushton (D) ${ }^{4}$
}

To cite: Punnoose A, Weiss 0 , Khanduja V, et al. Effectiveness of prehabilitation for patients undergoing orthopaedic surgery: protocol for a systematic review and meta-analysis. BMJ Open 2019:9:e031119. doi:10.1136/ bmjopen-2019-031119

- Prepublication history and additional material for this paper are available online. To view these files, please visit the journal online (http://dx.doi. org/10.1136/bmjopen-2019031119).

Received 16 April 2019 Revised 25 October 2019 Accepted 04 November 2019

A Check for updates

(c) Author(s) (or their employer(s)) 2019. Re-use permitted under CC BY-NC. No commercial re-use. See rights and permissions. Published by BMJ.

${ }^{1}$ Physiotherapy Department, Cambridge University Hospitals NHS Foundation Trust,

Cambridge, UK

${ }^{2}$ Institute of Clinical Sciences, University of Birmingham, Birmingham, UK

${ }^{3}$ Department of Trauma and Orthopaedics, Cambridge University Hospitals NHS Foundation Trust, Cambridge, UK ${ }^{4}$ School of Sport, Exercise and Rehabilitation Sciences, University of Birmingham Edgbaston campus, Birmingham, UK

Correspondence to Anuj Punnoose; anujpunnoose30@gmail.com

\section{ABSTRACT}

Introduction Undergoing major surgery can induce physical and functional decline. Prehabilitation programmes aim to improve physical fitness and function preoperatively and could enhance postoperative recovery and outcomes. Prehabilitation interventions have been utilised across a range of orthopaedic populations of all ages and can be multimodal in nature. The aim of this study is to evaluate the effectiveness of prehabilitation for patients undergoing orthopaedic surgery including day surgery procedures. It will also investigate the components of prehabilitation to understand optimum duration and frequency of programmes. Methods/design Systematic review and meta-analysis designed in accordance with PreferredReporting Items for Systematic Review and Meta-Analysis Protocols. A comprehensive electronic search will be performed in MEDLINE, CINAHL, AMED, Embase, PEDro and Cochrane CENTRAL databases in order to identify randomised control trials published between January 2000 to 25 March 2019. ISI Web of Science, System for information on grey literature and the European Union clinical trials registry will identify studies that are underway or unpublished. Two independent reviewers will carry out the searches, study selection (title and abstract and full text stages), data extraction, risk of bias assessment (Cochrane Risk of Bias tool 2.0) and evaluation of overall strength of evidence. Meta-analyses will be used for data which demonstrates homogeneity, otherwise a narrative synthesis will be performed for groups of studies of high heterogeneity $\left(\mathrm{I}^{2}\right.$ $>50 \%$ ). The overall strength of the body of evidence will be assessed using Grading of Recommendations Assessment, Development and Evaluation.

Ethics and dissemination This study raises no ethical issues. This study aims to identify the effectiveness of prehabilitation interventions and may assist clinicians in determining which components, duration, frequency and the method of delivery would form the most effective prehabilitation intervention for patients undergoing an orthopaedic surgical procedure. The findings will be disseminated through publication in a peer-reviewed journal and conference presentations.

PROSPERO registration number CRD42019123268.

\section{INTRODUCTION}

More than 8 million surgical procedures are performed in the UK each year. ${ }^{1}$ With an increase in the ageing population, it is
Strengths and limitations of this study

- This will be the first systematic review to include breadth of prehabilitation interventions prior to orthopaedic surgery including day surgery.

- The overall quality of evidence will be reported using Grading of Recommendations Assessment, Development and Evaluation.

- Anatomical variations of different joints, variations in surgical procedures and prehabilitation interventions may preclude meta-analysis and affect the overall level of evidence therefore not enabling firm conclusions of prehabilitation effectiveness to be established.

- Exclusion of non-English papers may lead to language bias.

anticipated that more surgical procedures will be performed in the future. ${ }^{2}$ Major surgery can induce pain, increase catabolism and oxygen demand that could lead to postoperative complications and deconditioning ${ }^{3}$ resulting in a decline of one's physical function. ${ }^{4}$ In order to withstand the stressor of inactivity following a surgical procedure, a period of prehabilitation prior to surgery may be beneficial in optimising physical and functional capacity in these patients. ${ }^{5}$ It is evident from the literature that individuals with limited physical fitness preoperatively have higher morbidity and mortality ${ }^{6} 7$ whereas individuals with better physical fitness are expected to have better postoperative outcomes. $^{8}$

The concept of prehabilitation was first mentioned in the literature in $1946 .{ }^{9}$ The authors reported how a period of physical and educational training along with nutritional support improved the physical and mental well-being of army recruits during the second world war. However, it was not until early 2000 , that researchers began to investigate the role of prehabilitation in improving 
postoperative outcomes. ${ }^{10}$ Since then several trials have been conducted to evaluate the benefits of prehabilitation in patients undergoing various surgical procedures ranging from cardiovascular to orthopaedics. ${ }^{11-14}$ Prehabilitation interventions have been utilised across all age groups ${ }^{15-18}$ and also in sports medicine as a tool to reduce the risk of sporting injuries. ${ }^{19}$ Although exercises are considered a key component in prehabilitation, the concept of prehabilitation interventions has evolved over the years becoming multimodal in nature. Interventions typically include exercise, nutrition, medical optimisation and psychological support delivered by a multidisciplinary team. $^{20-23}$

A number of good quality systematic reviews have been published recently investigating the benefits of prehabilitation within orthopaedics, but with varying conclusions. ${ }^{24-26}$ Cabilan $e t a l^{24}$ explored the effects of prehabilitation in all surgical patients including orthopaedics, cardiac and abdominal surgeries. The review found no significant benefit in postoperative function, pain and quality of life in patients who had joint arthroplasty for osteoarthritis, although there was some evidence that prehabilitation doses of $>500 \mathrm{~min}$ might reduce acute rehabilitation admissions (OR $0.51,95 \%$ CI 0.28 to 0.93 ). However, when participants who had undergone either a total hip or total knee replacement were grouped together, this was no longer significant. This review also reported that there was limited evidence on other surgical populations to derive any firm conclusions. The review had excluded day surgery patients and therefore has its limitations as there is a strong recent drive towards enhanced recovery programmes and more joint arthroplasties being performed as day cases. ${ }^{27-29}$ Additionally, the review only included trials published before March 2013.

Wang et $a l^{26}$ conducted a systematic review and metaanalysis on the effectiveness of prehabilitation for patients undergoing total hip arthroplasties (THA) and total knee arthroplasties (TKA) and reported slight improvement in: early postoperative pain (at 4 weeks), weighted mean difference -6.1 points, (95\% CI -10.6 to -1.6 points) on the Visual Analogue Scale ; function measured using the Western Ontario and McMaster Universities Arthritis Index (WOMAC) function score at 6 to 8 weeks -4.0 , (95\% CI -7.5 to -0.5$)$; time to climbing stairs -1.4 days, (95\% CI -1.9 to -0.8 days); toilet use -0.9 days, (95\% CI -1.3 to -0.5 days) and chair use -1.2 days, $(95 \% \mathrm{CI}-1.7$ to -0.8 days). However, the authors concluded that effects were too small and in the short-term to be considered clinically significant.

A more recent review by Moyer et $a l^{25}$ evaluated the benefits of prehabilitation in patients undergoing THA and TKA and reported significant improvements in quadriceps strength, pain, function and length of stay which contradicted previous reviews. Patients undergoing THA, had significantly less postoperative pain than controls (standard mean difference (SMD) $=0.15,95 \%$ CI 0.03 to $0.27, \mathrm{p}=0.017$ ). Postoperative function was also significantly improved compared with controls (THA $\mathrm{SMD}=0.32,95 \%$ CI 0.15 to $0.50, \mathrm{p}<0.001$; TKA $\mathrm{SMD}=0.32$, $95 \%$ CI 0.06 to $0.57, p=0.015$ ). Significantly greater quadriceps strength was observed after TKA $(\mathrm{SMD}=0.42$, $95 \%$ CI 0.16 to $0.68, p=0.002)$. Length of stay was significantly shorter after TKA (SMD $=0.54,95 \%$ CI 0.24 to 0.84 , $\mathrm{p}<0.001)$ and THA $(\mathrm{p}=0.027)$. This review included 35 studies which explored both preoperative exercises and patient education that may have had some influence on the positive results compared with previous reviews.

Previous reviews within the orthopaedic literature have mainly focused on the effectiveness of physical exercise, and on patients undergoing joint replacements rather than on arthroscopies or other day surgery procedures. Prehabilitation, in recent years has also become much broader encompassing multimodal interventions such as patient education and counselling, nutritional support alongside physical exercises. ${ }^{30}$ There is also limited evidence in the literature as to the most effective prehabilitation intervention and what components or combination of components, duration and frequency of prehabilitation intervention might be more effective. Therefore, the purpose of this study is to conduct an up to date, comprehensive systematic review and meta-analysis to explore the benefits of prehabilitation on postoperative outcomes for patients undergoing all types of orthopaedic surgical procedures including day case surgeries. This review will also encompass trials which utilised multimodal interventions in their prehabilitation programme.

\section{Objectives}

The primary objective is to synthesise the evidence on the effectiveness of prehabilitation in improving postoperative outcomes for patients undergoing orthopaedic surgery. Secondary objectives are to explore the core components of prehabilitation and whether there is evidence of an optimum duration and frequency that a patient needs to undergo prehabilitation in order to achieve better outcomes.

\section{METHODS}

We plan to conduct a systematic review of the literature according to a predefined protocol which complies with recommendations from the Cochrane Collaboration Musculoskeletal group ${ }^{31}$ and Centre of Reviews and Dissemination $^{32}$ guidelines and designed according to the Preferred Reporting Items for Systematic Reviews and Meta-Analysis (PRISMA-P) guidelines. ${ }^{33}$ and will be reported using the PRISMA-P checklist (online supplementary file 1 )

\section{Eligibility criteria}

- Trial design: Randomised controlled trials comparing prehabilitation (including multimodal prehabilitation interventions) to standard care.

- Participants: Adult participants (>18years) undergoing an orthopaedic surgical procedure. 
- Intervention: Prehabilitation or preoperative interventions including exercises/physiotherapy, patient education, pain management and anxiety reduction strategies.

- Comparators: Standard or usual care.

- Outcomes: Pain, muscle strength, function, healthrelatedquality of life (HRQoL) and disease specific/ joint specific outcomes.

- Language: All non-English publications will be excluded (at full text stage) to avoid introducing risk of bias.

- Publication: As the researchers only began investigating the effectiveness of prehabilitation on postoperative outcomes since the turn of the millennium, only trials published between January 2000 to 25 March 2019 would be included.

\section{Patient and public involvement}

Patients were not directly involved in the design of this study. As this is a protocol for a systematic review and no participant recruitment will take place, their involvement was not applicable.

\section{Information sources}

A comprehensive electronic search by two reviewers (AP and $\mathrm{OW}$ ) will be performed independently in MEDLINE (OVID), CINAHL (EBSCO), AMED (OVID), Embase, PEDro and Cochrane Register of Controlled Trials (CENTRAL) from January 2000 to 25 March 2019. We will also search the ISI Web of Science, System for information on grey literature and European Union clinical trials registry to identify studies that are underway or unpublished. In addition, a search for articles in press and published ahead of print will be conducted in relevant journals for orthopaedics (The Bone \& Joint journal, International Orthopaedics, Journal of Orthopaedics and Traumatology) and reference lists of included studies will be searched for further relevant studies.

\section{Search strategy}

The search strategy will include the intervention of interest. Medical subheading terms and keywords will include the following: Rehab*, prehab*, Physiotherapy, 'Physical therapy', Presurg*, Preoperat*, Prior, Before, Ahead, RCT and randomi*. See (online supplementary file 2) for an example of the search strategy for MEDLINE.

\section{STUDY RECORDS}

\section{Data management}

The specific bibliographic software EndNote will be used.

\section{Selection process}

Titles and abstracts (stage 1) followed by full-texts of potentially relevant studies (stage 2 ) will be independently screened by two reviewers (AP and OW). Where no abstract is available, full-text articles will be obtained and assessed for eligibility. Disagreements will be solved by discussion. Where no consensus can be reached, a third reviewer (AR) will be consulted. The process of study selection will be summarised using a Preferred Reporting Items for Systematic Reviews and Meta-Analyses flow diagram. ${ }^{33}$

\section{Data collection process and items}

Two reviewers will extract the data independently using a standardised form. A third reviewer will independently check the data for consistency and accuracy. Data extracted will include the following summary data: sample characteristics, sample size, outcomes of interest, duration and method of prehabilitation delivery (home, face-to-face, electronic means) and components of prehabilitation programme.

\section{Risk of bias in individual studies}

Risk of bias for each included trial will be independently assessed by two reviewers (AP and OW). The third reviewer (AR) will mediate in situations of disagreement. The Cochrane Risk of Bias tool $2.0^{34}$ will be used to assess internal validity of each of the included studies. The tool addresses specific domains that are assessed as 'high risk', 'low risk', or can express 'some concerns'. The tool was updated recently by the Cochrane Bias methods group and is structured into five domains: randomisation process, deviations from intended interventions, incomplete outcome data, measurement of the outcome and selective outcome reporting. Selective reporting in trials will be identified by comparing outcomes used in the finalised articles against registered protocols, or by comparing outcomes from the trial's published methods to the results sections, where no existing trial protocols are available.

\section{Outcome measures}

Outcomes of interest are pain (eg, Visual Analogue Scale), muscle strength (eg, quadriceps strength measured by manual or motor driven equipment), function (eg, Timed Up and Go), HRQoL (eg, EQ-5D) and disease specific/ joint specific outcome measures (eg, WOMAC). Health economic outcome measures such as length of stay, readmission rates and adverse events will also be reported as appropriate.

\section{Data synthesis}

Quantitative data will, where possible, be pooled in statistical meta-analysis using RevMan 5.3. Decisions for metaanalysis will consider Risk of Bias of individual studies and data will be pooled and considered for analysis within the same surgical procedure, population, interventions and consistencies in follow-up time points if possible. A random effects model will be used to measure effect sizes. ${ }^{35}$ Standard mean difference (continuous data) and their 95\% confidence will be calculated for analysis. Groups of studies where there is high heterogeneity measured by $\mathrm{I}^{2}$ value $\left(\mathrm{I}^{2}>50 \%\right),{ }^{36}$ a narrative synthesis will be performed including tables and figures to aid in data presentation where appropriate. Data will be analysed narratively according to the nature of the prehabilitation interventions. Additional analyses such as subgroup 
analysis and meta-regression would be used to report the factors that could explain the heterogeneity.

\section{Meta-bias(es)}

The studies will be assessed for publication bias and selective reporting of outcomes. A search will be conducted in the grey literature and also conference proceedings for national and international conferences in the past 2 years to identify related and unpublished studies. Where insufficient data are available, attempts will be made to contact the authors for further information and will be reported clearly. We will also scrutinise all eligible studies as to whether intended outcome measures were actually reported in the studies. Funnel plots will be used to assess publication and small sample size bias where metaanalysis includes more than 10 trials.

\section{Confidence in cumulative evidence}

The overall strength of the body of evidence will be assessed using Grading of Recommendations Assessment, Development and Evaluation. ${ }^{37}$ The tool is structured into five domains: risk of bias, imprecision, inconsistency, indirectness and publication bias. Each important outcome will be assessed for the overall certainty in the evidence using three categories: high, moderate, low and/or very low and the authors have the option of decreasing their level of certainty by one or two levels (eg, from high to moderate). Finally, the overall strength of recommendations will be categorised as weak or strong.

\section{DISCUSSION}

To the best of our knowledge, this is the first systematic review to include the breadth of prehabilitation interventions prior to orthopaedic surgery including day surgery procedures. These findings may provide evidence to support clinicians in determining if prehabilitation interventions are effective and what components and delivery method is most effective in achieving better outcomes for these patients. This review will also inform further research investigating the effectiveness and the nature of prehabilitation. The Cochrane Risk of Bias tool 2.0 $0^{34}$ will be used to assess internal validity of each of the included studies and the overall quality of evidence will be reported using Grading of Recommendations Assessment, Development and Evaluation. ${ }^{37}$ In order to maintain the strength and quality of our recommendations it was deemed appropriate to limit the review to randomised control trials (RCTs) as RCTs are considered to offer the highest quality of evidence. ${ }^{38}$ A limitation of this systematic review is the exclusion of non-English studies, which may mean that some trials conducted in non-English speaking countries may have been omitted.

\section{Ethics and dissemination}

This study raises no ethical issues. On completion of this systematic review, the findings will be presented to clinicians and academics at national or international conferences relating to orthopaedics. We will also publish the results in peer-reviewed academic journals to reach clinical and academic experts in this field.

\section{Twitter Alison B Rushton @abrushton}

Acknowledgements The authors would like to thank Veronica Phillips, Assistant Librarian, University of Cambridge Medical Library for assisting in the literature search.

Contributors VK developed the idea and planning for the review. AP is leading the protocol development and dissemination. AP and OW are the first and second reviewers. $A R$ is the third reviewer. $A R$ and $V K$ ensured review quality. $A P, V K$ and $A R$ have contributed to the design and development of the protocol and all authors will contribute to data interpretation and manuscript draft. VK is the guarantor of the review. All authors have approved this final manuscript.

Funding This systematic review is conducted as a part of Health Education England funded Masters to PhD Bridging Program at the University of Birmingham. Competing interests None declared.

Patient consent for publication Not required.

Provenance and peer review Not commissioned; externally peer reviewed.

Data availability statement There are no data in this work.

Open access This is an open access article distributed in accordance with the Creative Commons Attribution Non Commercial (CC BY-NC 4.0) license, which permits others to distribute, remix, adapt, build upon this work non-commercially, and license their derivative works on different terms, provided the original work is properly cited, appropriate credit is given, any changes made indicated, and the use is non-commercial. See: http://creativecommons.org/licenses/by-nc/4.0/.

\section{ORCID iDs}

Anuj Punnoose http://orcid.org/0000-0003-4477-2822

Alison B Rushton http://orcid.org/0000-0001-8114-7669

\section{REFERENCES}

1 Abbott TEF, Fowler AJ, Dobbs TD, et al. Frequency of surgical treatment and related Hospital procedures in the UK: a national ecological study using Hospital episode statistics. Br J Anaesth 2017;119:249-57.

2 Etzioni DA, Liu JH, Maggard MA, et al. The aging population and its impact on the surgery workforce. Ann Surg 2003;238:170-7.

3 Wynter-Blyth V, Moorthy K. Prehabilitation: preparing patients for surgery. BMJ 2017;358.

4 Hoogeboom TJ, Dronkers JJ, Hulzebos EHJ, et al. Merits of exercise therapy before and after major surgery. Curr Opin Anaesthesiol 2014;27:161-6.

5 Ditmyer MM, Topp R, Pifer M. Prehabilitation in preparation for orthopaedic surgery. Orthopaedic Nursing 2002;21:43-54.

6 Ekelund U, Steene-Johannessen J, Brown WJ, et al. Does physical activity attenuate, or even eliminate, the detrimental association of sitting time with mortality? A harmonised meta-analysis of data from more than 1 million men and women. The Lancet 2016;388:1302-10.

7 Jack S, West M, Grocott MPW. Perioperative exercise training in elderly subjects. Best Pract Res Clin Anaesthesiol 2011;25:461-72.

8 Fortin PR, Clarke AE, Joseph L, et al. Outcomes of total hip and knee replacement: preoperative functional status predicts outcomes at six months after surgery. Arthritis Rheum 1999;42:1722-8.

9 PREHABILITATION. Prehabilitation, rehabilitation, and revocation in the army. Br Med J 1946;1:192-7.

10 Banugo P, Amoako D. Prehabilitation. BJA Educ 2017;17:401-5.

11 Howard R, Yin YS, McCandless L, et al. Taking control of your surgery: impact of a prehabilitation program on major abdominal surgery. J Am Coll Surg 2019;228:72-80.

12 Marmelo F, Rocha V, Moreira-Gonçalves D. The impact of prehabilitation on post-surgical complications in patients undergoing non-urgent cardiovascular surgical intervention: systematic review and meta-analysis. Eur J Prev Cardiol 2018;25:404-17.

13 Silver JK. Cancer rehabilitation and prehabilitation may reduce disability and early retirement. Cancer 2014;120:2072-6.

14 Swank AM, Kachelman JB, Bibeau W, et al. Prehabilitation before total knee arthroplasty increases strength and function in older adults with severe osteoarthritis. J Strength Cond Res 2011;25:318-25.

15 Bruns ERJ, van den Heuvel B, Buskens CJ, et al. The effects of physical prehabilitation in elderly patients undergoing colorectal surgery: a systematic review. Colorectal Dis 2016;18:0267-77. 
16 Gill TM, Baker DI, Gottschalk M, et al. A prehabilitation program for physically frail community-living older persons. Arch Phys Med Rehabil 2003;84:394-404.

17 Punnoose A, Gallagher C, Matthews J, et al. Rehabilitation of a national Taekwondo player following arthroscopic hip surgery: successfully achieving a return to gold medal winning performance levels. J Nov Physiother 2015;5.

18 Shaarani SR, O'Hare C, Quinn A, et al. Effect of prehabilitation on the outcome of anterior cruciate ligament reconstruction. Am J Sports Med 2013;41:2117-27.

19 Sewry N, Verhagen E, Lambert M, et al. Exercise-Based interventions for injury prevention in tackle collision ball sports: a systematic review. Sports Med 2017;47:1847-57.

20 Bolshinsky V, Li MH-G, Ismail H, et al. Multimodal prehabilitation programs as a bundle of care in gastrointestinal cancer surgery: a systematic review. Dis Colon Rectum 2018;61:124-38.

21 Bousquet-Dion G, Awasthi R, Loiselle Sarah-Ėve, et al. Evaluation of supervised multimodal prehabilitation programme in cancer patients undergoing colorectal resection: a randomized control trial. Acta Oncol 2018;57:849-59.

22 Minnella EM, Awasthi R, Gillis C, et al. Patients with poor baseline walking capacity are most likely to improve their functional status with multimodal prehabilitation. Surgery 2016;160:1070-9.

23 West MA, Wischmeyer PE, Grocott MPW. Prehabilitation and nutritional support to improve perioperative outcomes. Curr Anesthesiol Rep 2017;7:340-9.

24 Cabilan CJ, Hines S, Munday J. The effectiveness of prehabilitation or preoperative exercise for surgical patients: a systematic review. JBI Database System Rev Implement Rep 2015;13:146-87.

25 Moyer R, Ikert K, Long K, et al. The value of preoperative exercise and education for patients undergoing total hip and knee arthroplasty: a systematic review and meta-analysis. JBJS Rev 2017;5:e2.

26 Wang L, Lee M, Zhang Z, et al. Does preoperative rehabilitation for patients planning to undergo joint replacement surgery improve outcomes? A systematic review and meta-analysis of randomised controlled trials. BMJ Open 2016;6:e009857.

27 Cross MB, Berger R. Feasibility and safety of performing outpatient unicompartmental knee arthroplasty. Int Orthop 2014;38:443-7.

28 Dorr LD, Thomas DJ, Zhu J, et al. Outpatient total hip arthroplasty. J Arthroplasty 2010;25:501-6.

29 Jenkins C, Jackson W, Bottomley N, et al. Introduction of an innovative day surgery pathway for unicompartmental knee replacement: no need for early knee flexion. Physiotherapy 2019;105:46-52.

30 Le Roy B, Selvy M, Slim K. The concept of prehabilitation: what the surgeon needs to know? J Visc Surg 2016;153:109-12.

31 Ghogomu EAT, Maxwell LJ, Buchbinder R, et al. Updated method guidelines for Cochrane musculoskeletal group systematic reviews and metaanalyses. J Rheumatol 2014;41:194-205.

32 CRD. Systematic reviews:CRD's guidance for undertaking reviews in healthcare. 3rd ED. Center for Reviews and Dissemination (CRD): BMJ Publishing Group, 2009.

33 Moher D, Shamseer L, Clarke M, et al. Preferred reporting items for systematic review and meta-analysis protocols (PRISMA-P) 2015 statement. Syst Rev 2015;4:1.

34 Higgins J, Sterne J, Savović J, et al. A revised tool for assessing risk of bias in randomized trials. Cochrane Database of Systematic Reviews 2016.

35 Hedges L. A random effects model for effect sizes. US American Psychological Association; 1983: 388-95.

36 Higgins JPT, Thompson SG. Quantifying heterogeneity in a metaanalysis. Stat Med 2002;21:1539-58.

37 Guyatt G, Oxman AD, Akl EA, et al. Grade guidelines: 1. IntroductionGRADE evidence profiles and summary of findings tables. J Clin Epidemiol 2011;64:383-94.

38 Guyatt G, Rennie D, Meade M, et al. Users' guides to the medical literature. 2nd ed. New York: McGraw Hill Medical, 2008. 